\title{
INTEGRATED MICRO-LIQUID DOSING SYSTEM
}

\author{
Theo S.J. Lammerink, Miko Elwenspoek and Jan H.J. Fluitman \\ MESA Research Institute, University of Twente, P.O. Box 217, \\ 7500 AE Enschede, the Netherlands.
}

\begin{abstract}
An integrated micro-liquid dosing system consisting of a micro-pump and a micro-liquid flow sensor is demonstrated. The dosing system allows accurate dosing of liquid in the $\mu \mathrm{l}$ regime and can easily be integrated with components as mixers and detectors to micro-liquid handling systems [1].

The liquid pump is of the reciprocating type with a thermo-pneumatic actuator. The micro-liquid flow sensor is based on the thermal anemometer type. Both pump and flow-sensor are realised in a 3-inch (100)- Si wafer using a $\mathrm{KOH}$ bulk etch from both sides of the wafer.

Due to its small heat capacity, the flow sensor has a small time constant, making it suitable for the function of measuring the liquid flow. The effective liquid dose is calculated by integration of the flow sensor signal. The pumped flow is in the range of $0-50 \mu \mathrm{l} / \mathrm{min}$. The system can simply be expanded with other micro-liquid handling components such as mixers detectors etc.
\end{abstract}

\section{INTRODUCTION}

Recently there is a growing interest in the research on micro-liquid handling systems $[1,2]$. Basic components in liquid handling systems are pumps and flow sensors. Pumps and flow sensors have already been realised separately, see e.g. $[3,4,5]$. The combination of these components needs hybrid assemblies, conventional use of tubes etc. Advantages of integrating the components are: small amount of liquid in the system and the possibility of realising complex liquid handling functions in a batch process. The combination of the pump and flow sensor in combination with a mixer [6] and detector can be exploited in more complex chemical analysing systems (see fig. 1).

We propose a closed loop concept for a liquid dosing system and we demonstrate the integration of a liquid pump and a liquid flow sensor. Demands for both the pump and the flow sensor are the capability of operating in the $\mu \mathrm{l} / \mathrm{min}$ range and a compatible fabrication process suitable for integration on a single wafer.

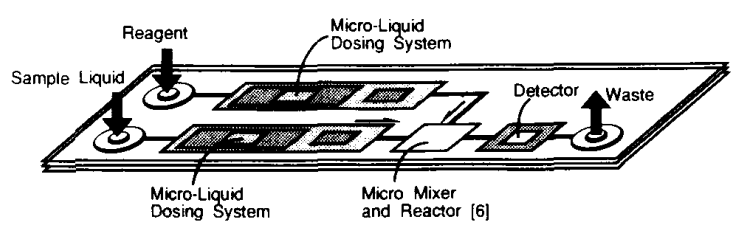

Fig. 1. Basic concept of Micro-Liquid Handling System [1].

\section{DOSING SYSTEM}

For a correct and reliable dosing of a certain volume of liquid it is necessary that the yield of a pump is independent of the pressure difference over that pump. Different pump principles are presented $[3,4]$, however they are not suitable to directly perform the dose-function of their own. In combination with a flow sensor, and an electronic circuit it is relative easy to control the yield (see fig. 2). The signal of the flow sensor has to be integrated and the speed and accuracy of the sensor have to be sufficient in order to lead to an accurate control of the dose.

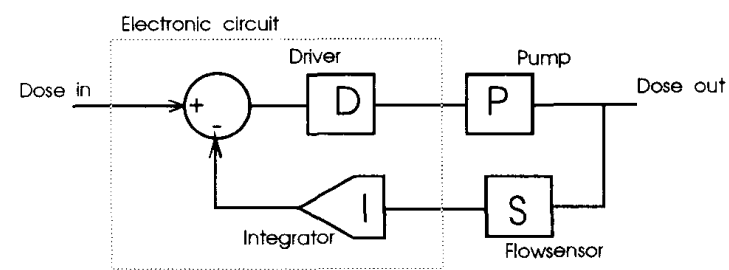

Fig. 2. Dose Control Loop. The liquid volumes moved by the pump are controlled by a flow sensor and an electronic circuit. 


\section{PRINCIPLE OF PUMP}

The reciprocating pump has two passive valves and a pump chamber. It is shown in fig. 3. The pump-actuator is thermo-pneumatic. In the air-chamber, the air is periodically heated by electrical dissipation in the heater resistor. Due to the varying temperature, the air-pressure in the chamber will periodically deflect the pump membrane, which results in the transport of liquid through the pump.

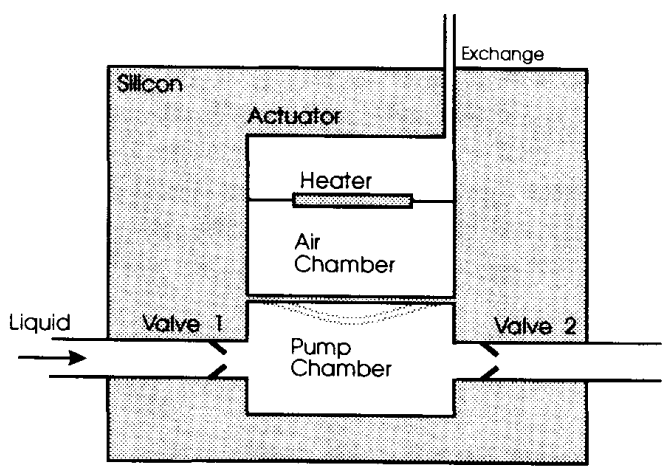

Fig. 3. Pump principle of the thermo-pneumatic reciprocating liquid pump.

\section{MODEL OF PUMP}

The model of the pump in the dosing system is similar to the model for the earlier developed thermo-pneumatic liquid pump [3]. The pump is modelled with the bondgraph method which is very convenient if several physical domains are involved [7]. The pump is devised into three (energetically coupled) sub-systems: a thermal subsystem, a pneumatic sub-system and a hydraulic subsystem.

The thermal sub-system consists of the heater, its (heat conducting) mechanical support and the (ideal) gas in the air-chamber. The heat capacity of the gas is neglected relative to that of the heater resistor. The lumped element model of the thermal sub-system consists of heat conducting and heat capacitance elements. With the materials and the heater used and air-chamber geometry's, the 'thermal' relaxation is calculated: $\tau_{t h}=$ $0.05 \mathrm{~s}$. The gas in the chamber reaches is steady state temperature within fractions of a second.

The pneumatic sub-system consists of the gas in the airchamber in combination with the exchange channel with the surroundings and the (movable) pump membrane. The sub-system can be described (in approximation, since the pneumatic system is non-linear) by a 'pneumatic' relaxation time, which is determined by the volume of the air-chamber and the flow resistance of the exchange channel. For the pump described here, $\tau_{p}=30 \mathrm{~s}$.
The hydraulic sub-system consists of the liquid (with its density and dynamic viscosity) in combination with the pump-chamber, the valves, the liquid channels, the flow sensor and the in- and outlet tubes to the dosing system. Since the valves show a highly non-linear behaviour, the hydraulic system cannot be described with a single 'hydraulic' relaxation time. However simulations for the dosing system described here, show a hydraulic relaxation time $\tau_{h}$ in the order of $1 \mathrm{~s}$. The pump functions properly if $\tau_{h}<\tau_{p}$, since otherwise the expanding gas only would display the air in the air-chamber. Interestingly, the dynamics of the pump is limited by the hydraulics and not by the thermal actuation.

The valves implemented are of the normally closed type, which means that the pressure changes in the airchamber have to be higher than the threshold pressure for the valves.

\section{PRINCIPLE OF FLOW SENSOR}

Fig. 4. illustrates the principle of the flow sensor. Three resistors are located in the middle of a flow channel. Heat is dissipated in the middle resistor called the heater $(\mathrm{H})$. The resulting temperature distribution is sensed with two temperature sensitive resistors $\mathrm{T}_{1}, \mathrm{~T}_{2}$ located symmetrically up- and downstream with respect to the heater. The heater as well as the sensing resistors are placed on supporting beams which cross the flow channel in the $x-y$ plane.

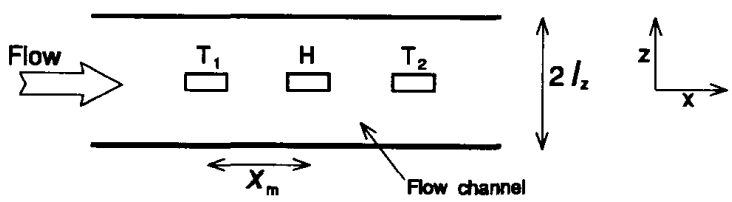

Fig. 4. The liquid flow is measured by its influence on the temperature distribution in the sensor resulting from heat generation in the heater $H . T_{1}$ (upstream) and $T_{2}$ (downstream) are temperature sensors.

At zero flow rate, no convection exists in the flow channel, and the heat generated by the heater will be transferred only by the axial and tangential diffusion through the fluid and by the conduction through the heater and sensor support to the flow channel walls.

- constant power mode

In this mode, the dissipated heat in the heater $\mathrm{H}$ is kept constant and the temperature difference $T_{2}-T_{1}$ is an accurate measure for the flow.

- constant temperature mode

In this mode the temperature of the heater is directly measured and kept constant. The electrical power needed is a measure of the flow. In this mode the flow sensor is very fast. 


\section{MODEL OF FLOW SENSOR}

The model for the flow sensor is extensively described elsewhere [5]. Here only a brief description will be given. The temperature profile in the $y$ and $z$ direction (see fig. 4) is assumed to be constant resp. linear. With $v$ the liquid velocity the temperature distribution in the middle of the channel can be described with:

$D \cdot \frac{\partial^{2} T}{\partial x^{2}}-v \cdot \frac{\partial T}{\partial x}-g \cdot D \cdot T=0$

with $D=\kappa / \rho c$ the thermal diffusivity of the liquid ( $\kappa$ the thermal conductivity, $\rho$ the density, $c$ the heat capacity (at constant pressure) ) and $g=1 / l_{z}^{2}$. Eqn. (1) is linear in T and can be solved analytically. The temperature distribution for three different flow velocities is given in fig. 5.

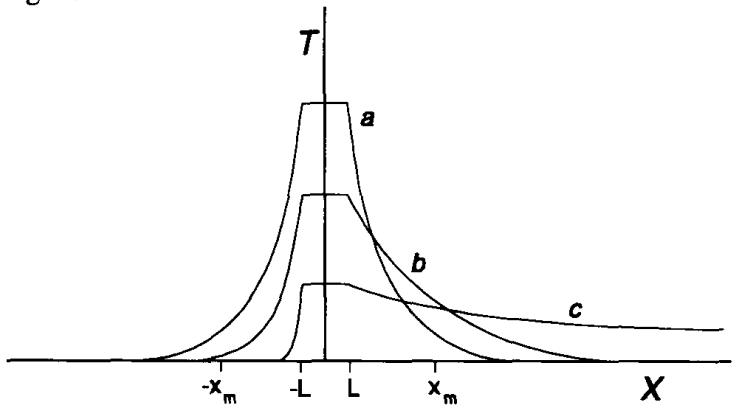

Fig. 5. Temperature distribution in the middle of the channel as a function of the position $x$. The heater is from $x=$ $L$ to $x=L . a$ ) $v=0$; b) $v=0.001$; c) $v=0.01$.

The temperature at the two sensor sites, upstream and downstream, the heater temperature $T_{h}$ and the temperature difference $\Delta T$ are given in fig. 6 .

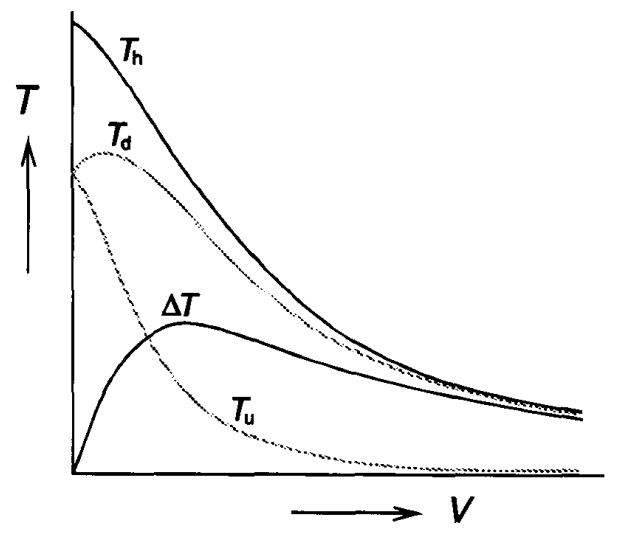

Fig. 6. Flow sensor temperatures as function of the flow velocity $v . T_{h}$ is the heater temperature, $T_{u}, T_{d}$ the upstream resp. downstream sensor temperature and $\Delta T$ the temperature difference between the downstream sensor and upstream sensor.
- liquid dependence

The sensor characteristics are strongly influenced by the thermal diffusivity $D$ of the liquid involved. The sensitivity of the sensor $S=\Delta T / v$, for low velocities $(v \rightarrow 0)$ is given by [5]:

$S=0.092 \cdot \frac{l_{z}}{l_{y}} \cdot \frac{P}{\kappa \cdot D}$

with $P$ the dissipated power in the heater, $\kappa$ the liquid heat conductivity, $l_{y}$ the channel width, $x_{m}$ the measurement distance, $l_{z}$ half the channel height. Sensing water-flow with $l_{y}=1000 \mu \mathrm{m}, l_{z}=250 \mu \mathrm{m}, \kappa=0.6$ $\mathrm{W} / \mathrm{mK}, D=1.410^{-7} \mathrm{~m}^{2} / \mathrm{s}$ and $10 \mathrm{~mW}$ dissipation in the heater we find $S=2.7 \mathrm{~K} /(\mathrm{mm} / \mathrm{s})$. For water and the given channel geometry, the temperature difference $\Delta \mathrm{T}$ is maximal for $v=11 \mathrm{~mm} / \mathrm{s}$. A liquid like iso-propanol has $D=6.96 \cdot 10^{-8} \mathrm{~m}^{2} / \mathrm{s}$ and a maximal $\Delta \mathrm{T}$ for $v=6 \mathrm{~mm} / \mathrm{s}$.

\section{FABRICATION}

The realisation process of the dosing system is shown in fig. 7. On a double sided polished $380 \mu \mathrm{m}<100>\mathrm{Si}$ wafer alignment marks, $3 \mu \mathrm{m}$ deep valleys (RIE etch, explained later) and $\mathrm{SiO}_{2}$ valve ridges are deposited and patterned (see 1,2 in fig. 7). The $\mathrm{SiO}_{2}$ valve ridges are used for selective bonding. Then a $1 \mu \mathrm{m}$ LPCVD SiN layer is deposited and patterned to form the mechanical support for the resistors of the pump and the flow sensor resistors (see 3 in fig. 7). The deposition parameters are such that there is no mechanical stress in the SiN layer. Next, the SiN layer on the top surface is patterned with the mask needed to etch anisotropically the cavity beneath the heater in the air-chamber of the pump, the top of the valves and for the liquid channels. The latter are aligned parallel to the $\langle 110\rangle$ crystal direction. The SiN supportbeams across the channel (flow sensor) and the cavity (under pump heater) have a width of $40 \mu \mathrm{m}$ and are oriented in the $<100\rangle$ crystal direction. The beams cross each other at a hart-to- hart distance of $200 \mu \mathrm{m}$. On the bottom surface, the SiN layer is patterned with the mask for the liquid channels, the valves and the pump chamber. Also a photoresist mask for the via's in the Si-wafer (e.g. needed for the 'hole' in the inner ridges of the valves) is applied. On the top-surface, a $200 \mathrm{~nm} \mathrm{CrAu}$ layer for the resistors is deposited and patterned ('lift-off', see 4 in fig. 7). The $30 \mathrm{~nm}$ Chromium layer is applied for optimal adhesion of the Au layer. At the bottom-surface, the wafer is $40 \mu \mathrm{m}$ deep dry etched (RIE; only the 'via'-mask active at the bottom-surface). Now the SiN mask at the bottomsurface is made active (RIE strip of photoresist) and the wafer is etched through (see 6 in fig. 7). The cross section of the liquid channels is an inverse trapezoid which is $1000 \mu \mathrm{m}$ wide at the top and is $170 \mu \mathrm{m}$ deep. The cavities in the top Pyrex wafer (G1 in fig. 7) are isotropically 
etched in a $50 \%$ HF solution at room temperature with a sputtered silicon layer as etch mask. The feed-throughs $(\phi$ $=400 \mu \mathrm{m}$ ) for the electrical contacts (in G1) and in and outlet (in G2) are made by drilling. Finally both Pyrex wafers and the structured Si wafer are anodically bonded $\left(1000 \mathrm{~V}, 450^{\circ} \mathrm{C}\right)$ to each other. A cross section of the completed dosing system is given in fig. 8 .

1

2

3

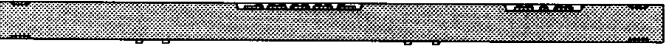

5

6

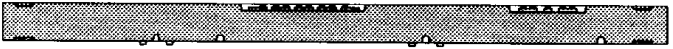

G1

G2
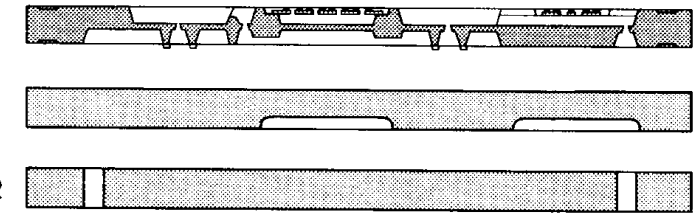

Fig. 7. Process sequence for the dosing system.

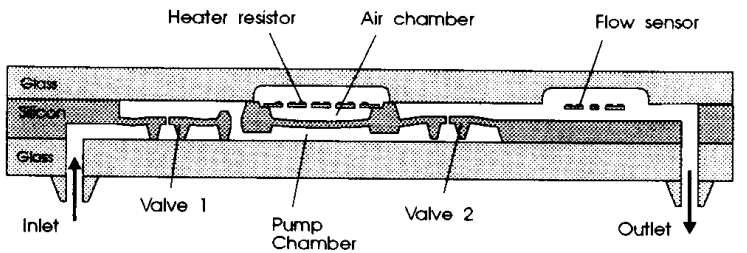

Fig. 8. Cross section of dosing system according line $A-A^{\prime}$ in fig. 10.

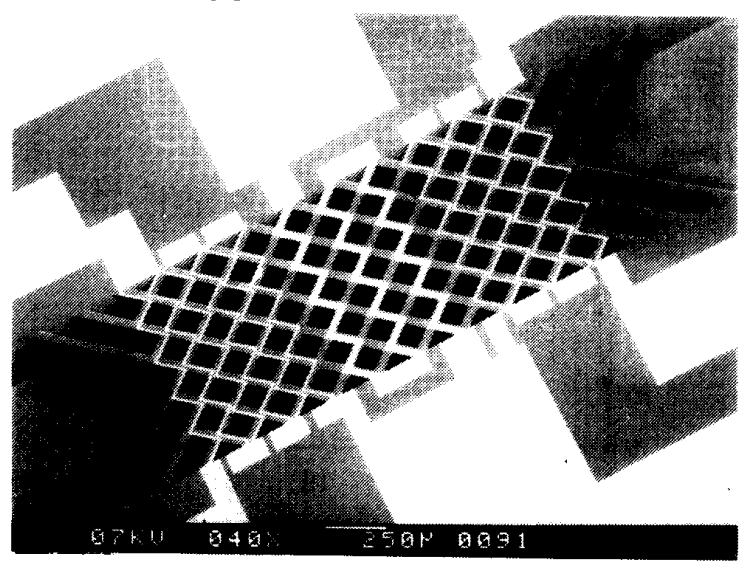

Fig. 9. SEM photograph of flow sensor. The channel width is $1 \mathrm{~mm}$.

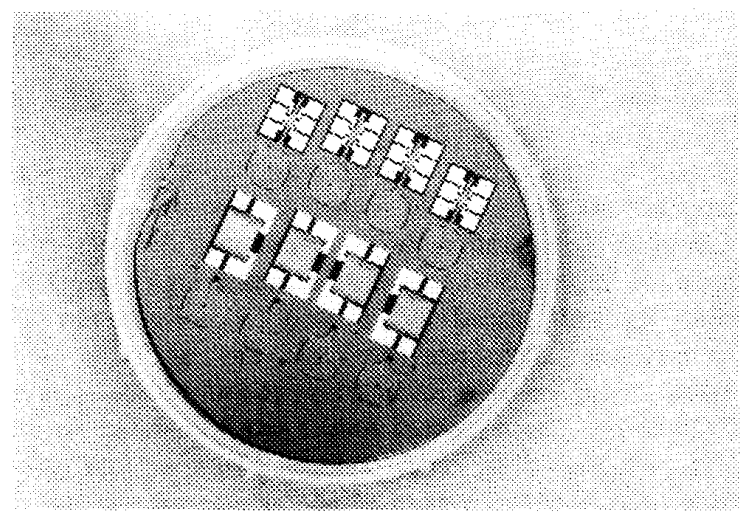

Fig. 10. Photograph of the Si-wafer with four dosing systems next to each other. The glass wafers are not applied yet.

The pump-heater resistor and the flow-sensor resistors are wired according fig. 11. Additional contacts are implemented to omit the influences of the bond-pad series-resistance's on the resistance measurements. Sketches of the liquid in- and outlet and of the electrical feed-throughs are given in fig. 12.
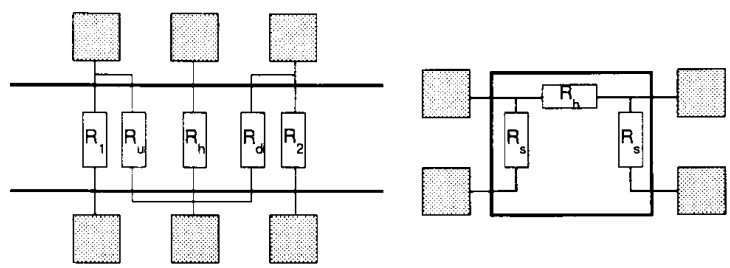

Fig $11 . \quad$ a) Flow sensor wiring. $R_{u}, R_{d}$ the sensing resistors upstream resp. downstream. $R_{h}$ the heating resistor. Resistors $R_{I}$ and $R_{2}$ are implemented in order to obtain a "4-point" measurement avoiding parasitic series resistances. b) Pump heater wiring; $R_{h}$ covers the pump-membrane area: $7 \times 7 \mathrm{~mm}^{2}$.
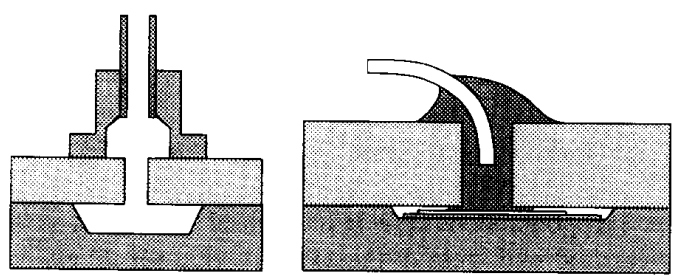

Fig 12. Sketch of liquid inlet and outlet and of the electrical feed-trough.

\section{- priming}

The dosing system is not self-priming. However it showed that it can be filled straightforwardly by injecting IPA in the inlet using a syringe. Filling the system with water is accomplished by first filling it with IPA and then switching over to water. 


\section{EXPERIMENTS}

First the flow sensors are characterised. This is done by forcing a flow in the forward direction through the flow sensor by means of a syringe pump. The flow sensors operate in the accurate 'constant power' mode. The electrical configuration for the read-out of the sensors is given in fig. 13. The temperature measuring resistors $R_{u}$ and $R_{d}$ are placed in a bridge configuration. $A$ temperature difference between the upstream and downstream sensor is linear converted to a bridge-output voltage [5]. The measured sensor sensitivities are in the order of $25 \mu \mathrm{V} / \mathrm{K}$ for a heater dissipation of $10 \mathrm{~mW}$ using IPA as liquid. The bridge output voltage as function of the liquid flow is given in fig. 14.

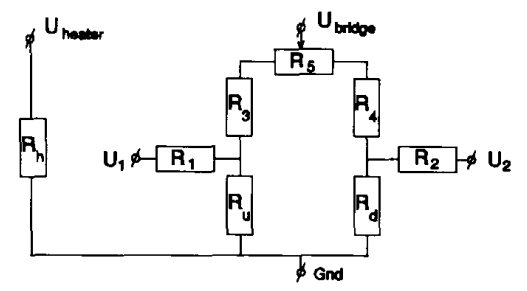

Fig. 13. Sensor measurement circuit for constant power mode. Nominal resistor values are: $R_{h}=800 \Omega ; R_{u}, R_{d}=$ $3300 \Omega ; R_{1}, R_{2}=725 \Omega ; R_{3}, R_{4}=3300 \Omega ; R_{5}=$ $100 \Omega[5]$.

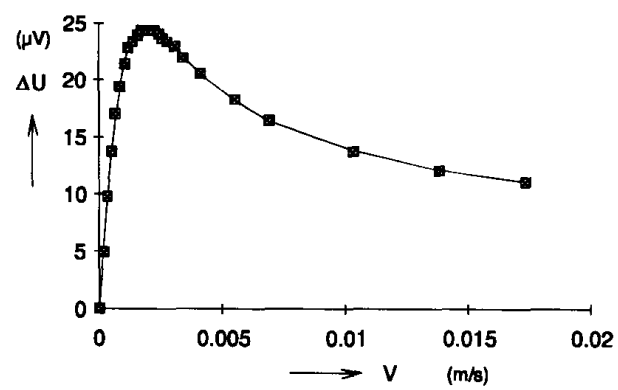

Fig. 14. Flow-sensor output with water as liquid. With a flow channel of $1000 \mu \mathrm{m} \times 500 \mu \mathrm{m}$, a flow velocity $v=20$ $\mathrm{mm} / \mathrm{s}$ is equal to a volume flow of $600 \mu \mathrm{l} / \mathrm{min}$. For water: $\kappa=0.6 \mathrm{~W} / \mathrm{mK}, D=1.410^{-7} \mathrm{~m}^{2} / \mathrm{s}[5]$.

Next the spontaneous flow behaviour of the dosing system is measured with help of the experimental set-up given in fig. 15. If the input pressure is higher then twice the valve 'pre-pressure', liquid will flow through the dosing system. When the syringe pump is programmed at a certain flow, both capillary tubes indicate the pressure difference over the dosing system.

The liquid pump is characterised with the help of a square wave generator for the pump-heater, a high speed digital voltage meter for measuring the flow sensor output. The results on the dynamic pump behaviour of the system are given in fig. 16. For a first characterisation of the pump, the pumping speed is measured with the help of a capillary tube at zero pressure difference between the inlet and the outlet of the dosing system. Results are given in fig. 17.

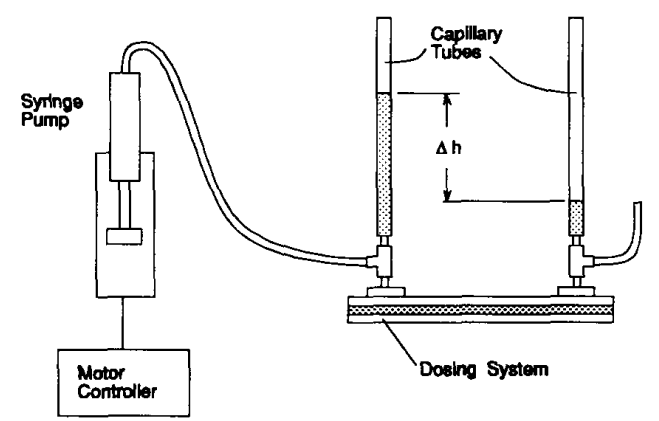

Fig 15. Experimental set-up for measuring the spontaneous flow and the pre-pressure of the normally closed valves.
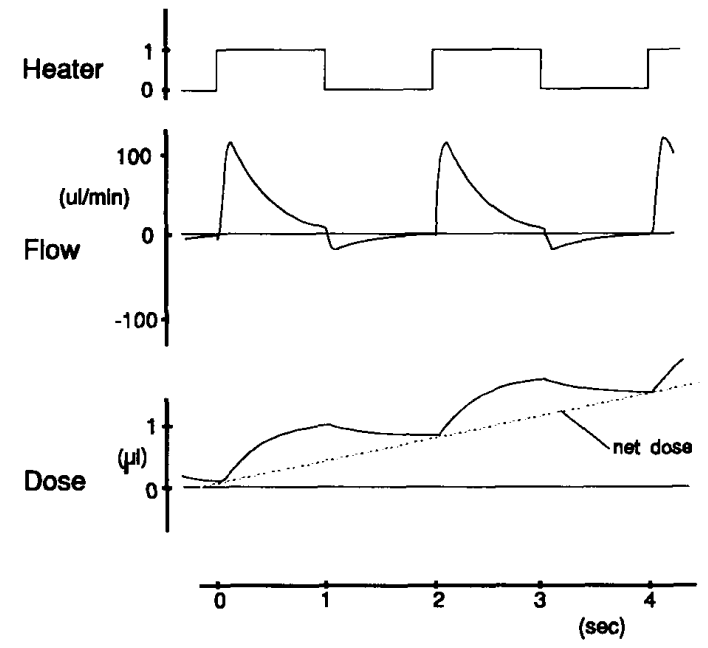

Fig. 16. Actuator-signal, measured liquid flow and the calculated (integration of flow) liquid dose as a function of time. The flow-sensor output is measured every $50 \mathrm{~ms}$.

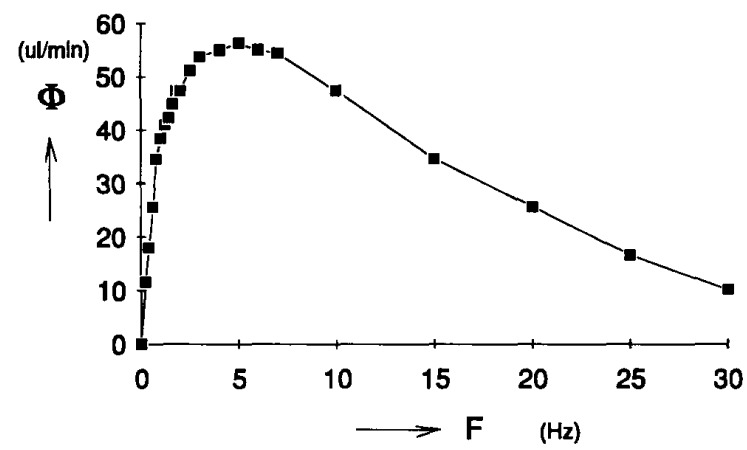

Fig. 17. Pumped liquid dose as function of the frequency of the pump-heater signal $(P=2.5 \mathrm{~W})$. 


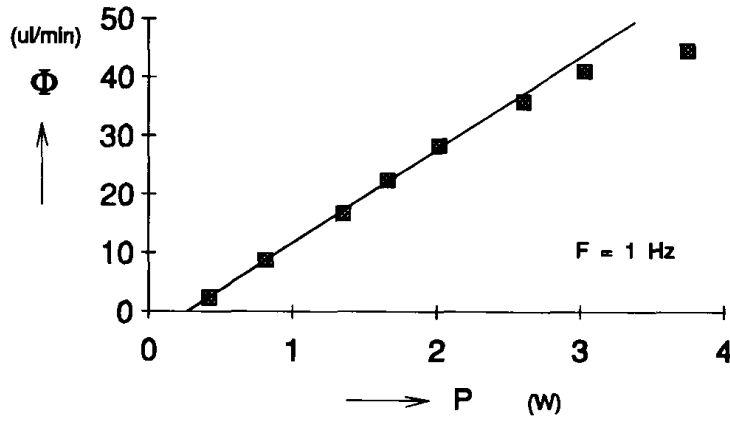

Fig. 18. Pump speed as a function of the applied heater power.

\section{DISCUSSION}

-flow sensor

The flow sensor is extensively discussed in [5]. The measurement result shown in fig. 14 is qualitatively similar to the model results shown in fig. 6 . For low flowvelocities the output signal is linear with $v$ and at a certain 'turn-over' flow velocity, the output signal reaches a maximum. For a reliable flow signal the sensor has to be used for flow velocities below $v_{t o}$. In the dosing system, this counts for the momentary flow-velocity.

- static properties of the pump

The passive valves are normally closed. The built-in pressure of the valves is proportional to the bending stiffness of the valve membranes.

For a dosing system described here, with $30 \mu \mathrm{m}$ valve membranes we measured spontaneous flow above 0.01 atm. For a thickness of about $40 \mathrm{~mm}$ we measured spontaneous flow above $0.03 \mathrm{~atm}$. These values are in agreement with the expected ones.

- dynamic properties of the pump

The 'thermal' relaxation time is measured using the temperature dependence of the pump-heater resistor. We measured $\tau_{t h} \approx 0.1 \mathrm{~s}$. With the pump-heater continuously activated, the 'pneumatic' relaxation is extimated from the pump-membrane displacement as a function of time: $t p=$ 15 s. Fig. 16 shows that the pump stroke with an input power of $5 \mathrm{~W}$ is about $0.9 \mu \mathrm{l}$. From the flow sensor signal we see that the hydraulic relaxation of the dosing system is within $1 \mathrm{~s}$. After a pump cycle some liquid flows back into the pump body (see also [3]).

At low pump frequencies the hydraulic system relaxes within one cycle, so the pump stroke is constant and the pump speed is linear with the pump frequency (see fig. 17). At higher frequencies there is not enough time for hydraulic relaxation and the yield decreases. At a fixed pump frequency, the pump speed is almost linear with the heating power $P$ (fig. 18). The offset in the curve is due to the back-flow or 'dead' volume of the pump. At high powers the curve is non linear.
- 'new' pump principle

The pressure built-up of the pump is limited by the maximum pressure built-up in the actuator chamber. With a liquid-vapour equilibrium the pressure can be increased dramatically, with comparable temperature deviations. This was experimentally verified with a pump with a broken pump membrane. With sufficient input power the alcohol that now penetrates the actuation chamber boils, giving rise to an actuation pressure large enough to pump against $1 / 3 \mathrm{~atm}$. This phenomenon will be discussed in greater detail elsewhere [9].

- concluding remarks

The integration of a liquid pump and a liquid flow-sensor into one integrated dosing system in a single Si-wafer has been demonstrated.

The predicted and measured pump characteristics as well as the flow sensor characteristics show a good agreement.

\section{ACKNOWLEDGEMENTS}

The authors would like to thank Erwin Berenschot, Dick Ekkelkamp, Rob Legtenberg and the staff of the MESAS\&A laboratory for their help in realising the samples and the experimental set-up.

\section{REFERENCES}

[1] F. C.M. v.d. Pol, "Micro Fluid Handling Systems", Micro Systems Technologies 90, 1990, pp. 799-805.

[2] A. Manz, N. Graber and H.M. Widmer, "Miniaturized Total Chemical Analysis Systems: a Novel Concept for Chemical Sensing", Sensors and Actuators, Bl (1990) pp. 244-248.

[3] F.C.M. v.d. Pol, H.T.G. van Lintel, M. Elwenspoek and J.H.J. Fluitman, "A Thermopneumatic Micropump based on Micro-engineering Techniques", Sensors and Actuators, A21-A23 (1990), pp. 198-202.

[4] A. Richter, A. Plettner, K.A. Hoffmann and H. Sandmayer, "A micromachined electrohydrodynamic (EHD) pump", Sensors and Actuators, A29 (1991) pp. 159-168.

[5] T.S.J. Lammerink, M. Elwenspoek and J.H.J. Fluitman, "Micro-Liquid Flow Sensor", Proc. Eurosensor VI conference, 4-10 october, San Sebastian, Spain, 1992.

[6] R.Miyake, T.S.J. Lammerink, M. Elwenspoek and J.H.J. Fluitman, "Micro Mixer with Fast Diffusion", Proc. MEMS '93 conference, Fort Lauderdale, FA, USA, 7-10 Feb. 1993.

[7] F.C.M. v.d. Pol, P.C. Breedveld and J.H.J. Fluitman, "Bond-graph modelling of an electro-thermo-pneumatic micropump", Techn. Digest MME '90, 2nd workshop on Micromachining, Micromechanics and Microsystems, (1990) pp. 19-24.

[8] C. Yang and H. Søeberg, Monolithic flow sensor for measuring millilitre per minute liquid flow, Sensors and Actuators A, 33 (1992) 143-153.

[9] T.S.J. Lammerink et al, submitted to Eurosensors VII Conference, 1993. 